\title{
Improving precipitation forecasts over Iran using a weighted average ensemble technique
}

\author{
Maede Fathi $^{1}$, Majid Azadi $^{2} *$, Gholamali Kamali ${ }^{1}$ and Amir Hussain Meshkatee ${ }^{1}$ \\ ${ }^{1}$ Department of Meteorology, Science and Research Branch, Islamic Azad University, Tehran, Iran. \\ ${ }^{2}$ Atmospheric Science and Meteorological Research Center, Tehran, Iran. \\ ${ }^{*}$ Corresponding author.e-mail: azadi68@hotmail.com
}

MS received 9 March 2018; revised 4 November 2018; accepted 25 November 2018; published online 13 May 2019

In this paper, the ensemble-weighted mean (ENSWM) technique is experimented for improving 24- to 72-hr precipitation forecasts over Iran during autumn and winter 2011 and 2012. The ensemble prediction system (EPS), used in this research, consists of nine different configurations of the weather research and forecasting model. In this technique, weights for each ensemble member at each grid point are assigned on the basis of the correlation coefficient (CC) between ensemble members and observed daily rainfall during a training period. Apart from ENSWM, precipitation forecasts using the simple ensemble mean (ENSM) are also generated and compared. Results showed that, in general, the forecast errors are relatively high along the coasts of the Caspian Sea in northern and at the Zagros mountainous areas located in western Iran. The skill of the rainfall forecasts of the ENSWM is examined against ENSM and individual members of the ensemble. The 24- to 72-hr forecasts are evaluated using common statistical scores including root mean-squared error (RMSE), and anomaly CC (ACC) for continuous forecasts and probability of detection (POD) score and threat score for categorical forecasts. The comparison reveals that the ENSWM is able to provide more accurate forecast of rainfall over Iran by taking the strength of each constituent member of the ensemble. It has been further found that the precipitation forecast skill of ENSWM is higher than ENSM and each ensemble member in the short-range time scale over Iran. The rainfall prediction skill over Iran was improved significantly using the weighted ENSWM technique. Results clearly show the advantage of using an EPS for the prediction of precipitation over the country vs. a single deterministic forecast for operational purposes. The RMSE of 24-, 48- and 72-hr forecasts in ENSWM relative to ENSM is reduced by 2, 2 and 5\%, respectively. The CC increased by $15 \%$ in the ENSWM relative to ENSM.

Keywords. Numerical weather prediction; ensemble forecast; ensemble-weighted mean (ENSWM) forecasting; simple ensemble mean (ENSM); rainfall prediction skill; WRF model.

\section{Introduction}

Since accurate weather forecasts are important for planning day-to-day activities in many areas such as hydrology and agriculture, they have attracted much attention in recent years. Timely and skillful precipitation forecasts are important for decision making when dealing with meteorological and hydrological hazards such as floods and droughts. Specifically, the reservoir operators can benefit from skillful precipitation forecasts for effective flood control, while during droughts farmers and water resource managers can also utilise precipitation forecasts to determine irrigation schedules 
for more effective drought mitigation. Hence, if the forecast is inaccurate, the consequences can be grave, both in terms of economic and social loss.

High-resolution quantitative precipitation forecasts using numerical weather prediction (NWP) models play an important role in the task of flood forecasting. The basis of the NWP models is to use numerical methods to solve the highly nonlinear thermodynamic and simplified dynamics equations governing the atmospheric motions. Since the atmosphere has a chaotic behaviour, it is impossible to find the exact solution of the equations and thus the obtained numerical solution is always contaminated by random errors. On the other hand, the differences between the real-world topography and land use, and their representations in the model led to systematic errors in the NWP model output. That is to say, the precipitation forecasts of the NWP models always suffer from errors in terms of the determination of their region and severity. Despite the fact that the quality of forecasts from NWP models has been improved in recent years, more accurate predictions of precipitation still remain a difficult and challenging task.

Epstein (1969) and Leith (1974), with the aim of improving forecasts, suggested that instead of using a definitive forecast, a collection of forecasts to be used, and this method of forecasting is called ensemble forecasting. An ensemble forecast is simply a collection of two or more forecasts verifying at the same time. Several methods are available to create NWP ensembles, accounting for the uncertainty in either the initial (Toth and Kalnay 1993, 1997; Buizza and Palmer 1995; Houtekamer et al. 1996; Molteni et al. 1996; Wang and Bishop 2003; Wang et al. 2004) and/or boundary conditions (Frogner and Iversen 2002; Romine et al. 2014) or the NWP model itself (Christensen et al. 2015). This study uses a weather research and forecasting (WRF) multi-physics ensemble that comprises nine model configurations with different cumulus, PBL, microphysics and parameterisation schemes of the surface layer. In order to improve the ensemble forecasting, statistical post-processing of the raw ensemble outputs is required to generate calibrated forecasts. Although ensemble forecasts are useful and provide more information than a single deterministic forecast, the main purpose of ensemble forecasting is the removal of the collective errors of multi-models (Durai and Bhardwaj 2013). The simplest method for issuing a probabilistic forecast using the ensemble prediction system output is the ensemble averaging method. The main problem of ensemble averaging method is that it assumes equal weight for all members and takes the simple average of all predictions, which may include several poor results. These poor models reduce the accuracy of averaged overall result. To overcome this problem of ensemble forecasting, Krishnamurti et al. (1999, 2000) introduced the multi-model ensemble (MME) technique, which showed a significant improvement in prediction skill. In the superensemble approach, the weight of each model is assigned based on the spatial and temporal performance. The strategy of the MME method consists of two phases: a training period and a forecast phase. In the first phase, the weight of each model is determined using the outputs from multi-models and their verifying corresponding observations. In the next phase, multi-model forecasts and statistics of the first phase led to the final superensemble forecast. Roy Bhowmik and Durai (2010, 2012) used a correlation coefficient (CC) method to find the weight of each member and then provided an MME forecast. They used the MME technique over India. Mitra et al. (2011) performed the experimental MME forecast of rainfall during 2009 monsoon. The difficulties in producing precipitation forecasts for smaller regions by the state-of-the-art global models are well known. The simple average of many models generally produces a higher skill score in comparison with each deterministic model. The skill of the forecast obtained by using the MME method is better than the skill of both simple ensemble mean (ENSM) and forecast of the deterministic models. Besides, neural network techniques are used in the MME method (Muller and Reinhardt 1991; Masters 1993) Mishra and Krishnamurti (2007) applied the super-ensemble method to the Indian monsoon, which increased the skill of the forecast using data from seven global models. In a report to evaluate the deterministic forecast in the United Kingdom Meteorological Office (UKMO) and the European Centre for MediumRange Weather Forecasts (ECMWF) ensemble, the advantages of the MME compared to the individual model data have been shown by Evans et al. (2000). Richardson (2001) used MME method to produce a deterministic and probabilistic forecast using four global models from the UKMO, German Meteorological Service, Meteo France and the National Centre for Environmental Prediction (NCEP). His results showed that the simple ENSM is a useful product. Ebert (2001) studied probabilistic precipitation forecasts and rainfall distribution using the 
multi-model data with seven global models for the Australian region. His initial tests confirmed that the ENSM precipitation forecast from these seven models has lower errors than the individual models. It was less clear whether an ensemble of only seven models could provide useful probabilistic rain forecasts.

Johnson and Swinbank (2009) used mid-range ECMWF, UKMO and NCEP/Global Forecast System (GFS) models to generate MME forecasts. In this forecast, bias correction, model-dependent weights and variance adjustments were investigated. The results showed that the MME gives a more skill forecast than a single forecast. More recent studies of monsoon rainfall have been reported using the MME method based on a simple linear regression approach (Roy Bhowmik and Durai 2008, 2010; Mitra et al. 2011). They did not mention the benefits of giving weights to member models over a simple ENSM. In other recent studies, e.g., Krishnamurti et al. (2009); Mitra et al. (2011) and Kumar et al. (2012), the MME forecast of monsoon rainfall for India resulted in the production of the lowest root mean-squared error (RMSE) and higher skill than the best model.

Due to the complex topography of Iran, timely and accurate precipitation forecast is a challenging task. The topography of our study area consists of rugged, mountainous rims surrounding high interior basins, leading in some cases to significant errors in the outputs of the NWP models. The intensity of the synoptic systems over the country depends on the polar front jet oscillations. Polar vortex strengthens in the winter and weakens in the summer due to its dependence on the temperature difference between the equator and the poles. One of the main causes of Iran's rainfall during winter is the migration of the polar jet stream towards lower latitudes approaching Iran. Except for a small coastal region of the Caspian Sea, located in northern Iran, the activity of precipitating synoptic systems over the country is mainly due to Rossby waves during winter. The north Indian Ocean, the Oman Sea and the Red Sea act as the main moisture sources of the synoptic systems during the winter season. In the coastal region of the Caspian Sea, the situation is completely different. While the moist air is approaching the country, Rossby waves progress moving from southwest to the northeast and have to move over the Zagros mountains (in western Iran) and cross the Alborz mountains (in northern Iran) and consequently loses much of its moisture content.
As a result, the humidity of the Indian Ocean, the Oman Sea and the Red Sea do not have a significant importance for the rainfall in the northern coastal regions of Iran. The Caspian Sea is the main source of moisture for the precipitating mesoscale and synoptic scale systems in its coastal region. In terms of water resource management, the precipitation maxima over the Caspian Sea coastal region have a great importance in the country. Thus, its accurate prediction has been always demanding. On the other hand, the small and mesoscale processes responsible for the highly complex patterns of precipitation in the region, such as the mountain-valley and the sea-land breezes are not generally well captured in NWP models, and thus the outputs of NWP models are not generally reliable in the coastal region of the Caspian Sea.

In the current study, an ensemble has been developed using different configurations of the WRF model, which uses nine different model configurations including the physical parameters of the cumulus, PBL, microphysics and the surface layer. They have been chosen according to some previous research studies. More discussions on the advantages of using the advanced multi-model ensemble approach could be found in some recent publications (e.g., Jaiswal et al. 2018) In this study, the socalled MME method, i.e., a weighted average of the ensemble members is applied for forecasting daily precipitation over Iran in the short-range time scale during winter of 2011-2012 and validated against the observations. The used verification indices are RMSE, anomaly correlation coefficient (ACC) as continuous indices, and probability of detection (POD) score and threat score (TS) for categorical forecasts.

The remaining part of this paper is organised as follows. Section 2 provides the definition of the applied methods. Section 3 reports the used data and also the application of the discussed methods. The verification and comparison results are presented in section 4, and finally, conclusions are drawn in section 5 .

\section{Methodology}

To generate the calibrated forecasts, the so-called ensemble-weighted mean (ENSWM) technique is used. In this technique, the weight given to each ensemble member is obtained by calculating the $\mathrm{CC}$ between the ensemble member forecasts and 
their corresponding observations in a training period (Durai and Bhardwaj 2013).

The ENSM forecast is calculated as

$$
\operatorname{ENSM}=\frac{1}{N} \sum_{k=1}^{N} F_{k}
$$

where $F_{k}$ is the $k$ th ensemble member forecast and $N$ is the total number of the ensemble members.

The number of points in the aforementioned regular grid is $230 \times 180$. Hence, the ENSWM forecast is generated by each ensemble member $(k)$ over all grid points $(i, j)$ using the equation

$$
\begin{aligned}
\operatorname{ENSWM}= & \frac{1}{N} \sum_{k=1}^{N} W_{i, j, k} F_{i, j, k}, \\
& i=1,2, \ldots, 230 ; \quad j=1,2, \ldots, 180,
\end{aligned}
$$

where $W_{i, j, k}$ is the weight given to the $k$ th ensemble member for each grid $(i, j)$. The weights are obtained from the following equation:

$$
W_{i, j, k}=\frac{C_{i, j, k}}{\sum_{k=1}^{N} C_{i, j, k}},
$$

where $C_{i, j, k}$ is the $\mathrm{CC}$ between the observation and forecast of precipitation for the grid $(i, j)$ of the $k$ th member.

For each member of the ensemble, the 24- to 72-hr forecast of the accumulated precipitation is extracted at a regular grid point with $0.1^{\circ}$ resolution covering Iran. In addition, the observation data at the synoptic stations are interpolated to the above-mentioned grid with gradient plus inverse-distance-squared (GIDS) method (Nalder and Wein 1998). The ENSWM method is used to generate calibrated forecasts in which the weight of each ensemble member is calculated using the CC (Durai and Bhardwaj 2013). The skill of ENSWM is compared with each member of ensemble and ENSM during the interested period. As previously mentioned, the GIDS method is used to interpolate the observation data from the synoptic stations to a regular grid. This method is based on multiple linear regression (MLR) of the data from a series of adjacent stations to estimate local slopes for each climate. In GIDS method, an MLR (equation 4) is fitted to data from a number of nearby weather stations:

$$
Y=a+m_{\mathrm{E}} E+m_{\mathrm{N}} \text { lat }+m_{\mathrm{W}} \text { lon, }
$$

where $Y$ is the value of a weather parameter, $E$ is elevation, lat is latitude and lon is longitude of the station; $a$ is a constant intercept and $m_{\mathrm{E}}, m_{\mathrm{N}}$ and $m_{\mathrm{W}}$ are the regional precipitation gradients for elevation, latitude and longitude, respectively.

The inverse of squared distance $\left(1 / d^{2}\right)$ between these $n$ nearby stations and unsampled locations are used as weights in the estimation of the weather parameter $\left(Y_{\mathrm{u}}\right)$ :

$$
Y_{\mathrm{u}}=\frac{\sum_{i=1}^{n} \frac{1}{d_{i}^{2}}\left(Y_{i}+m_{\mathrm{E}} \Delta E_{i}+m_{\mathrm{N}} \Delta N_{i}+m_{\mathrm{W}} \Delta W_{i}\right)}{\sum_{i=1}^{n} \frac{1}{d_{i}^{2}}},
$$

where $\Delta N, \Delta W$ and $\Delta E$ are the differences in latitude, longitude and elevation, respectively, between the unsampled location and a number of nearby weather stations.

Here, some performance skills are calculated to compare the ENSWM, ENSM and ensemble members against daily precipitation analysis at the regular grid points. The accumulated rainfall forecasts are verified using RMSE and ACC. In addition to the categorical verification method, the TS and POD using the forecasts and corresponding observations are calculated.

\section{Study area and data}

Iran has different topographical and climatic conditions, ranging from the mountainous areas in the northern and western Iran, the coastal zone of the Caspian Sea and the Persian Gulf, the forest lands above the northern barrier of the Alborz and the dry zone in the central part of the country. The study area covers the entire Iranian plateau area in southwest Asia. The complex topography and the climate of the Middle East and consequently Iran have important implications for synoptic systems. Iran is located to the southwest of Asia and its area is approximately $1,873,959 \mathrm{~km}^{2}$ and has a topography range of -26 to $5671 \mathrm{~m}$ a.s.l. (figure 1).

In this study, the forecast data are the accumulated 24- to 72-hr precipitation forecasts generated by the WRF-ARW model. Initialisation time is 12 UTC during the period from 1 September 2011 to 26 February 2012. The initial conditions for forecasts are provided by the GFS forecasts with $1^{\circ}$ horizontal resolution.

WRF is run with two nested domains. The large domain has a $45 \mathrm{~km}$ horizontal resolution and 


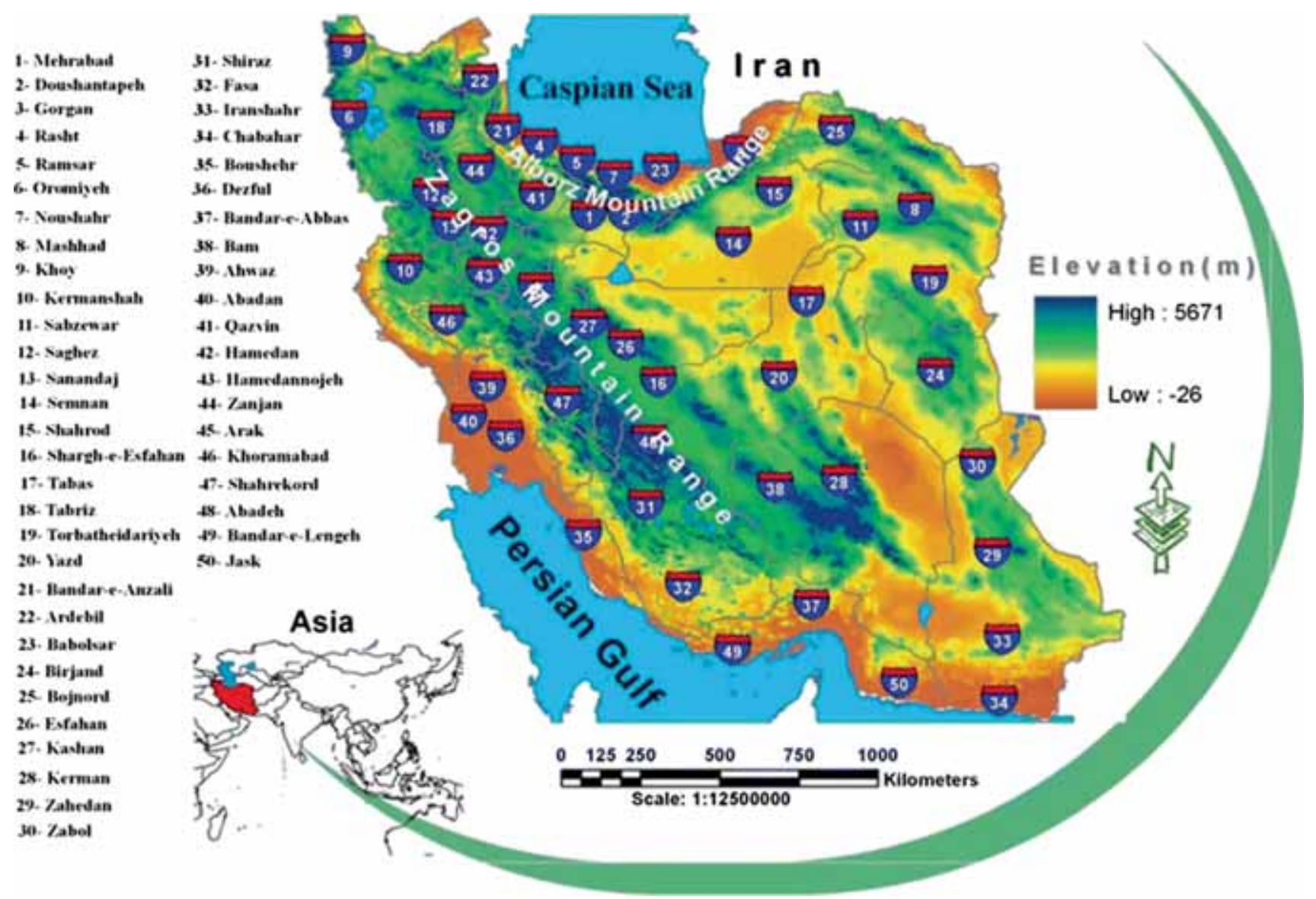

Figure 1. List and locations of the 50 weather stations utilised in this study and the topography of the country (Soltani et al. 2016).

covers an area in the southwest Middle East from $10^{\circ}$ to $51^{\circ}$ north and from $20^{\circ}$ to $80^{\circ}$ east. The small domain has a horizontal resolution of $15 \mathrm{~km}$ and covers an area between $23-41^{\circ}$ north and $42-65^{\circ}$ east (whole Iran). The WRF forecasts are interpolated to a regular grid with a horizontal resolution of $0.1^{\circ}$.

Observational data used in this study consist of the cumulative precipitation observations measured at 06:00 UTC in 306 irregularly spaced synoptic meteorological stations spread across Iran. In order to compare the precipitation forecasts with the corresponding observations, the observed 24-hr accumulated precipitation is interpolated to a regular grid using the so-called GIDS (Nalder and Wein 1998) method.

To assess the performance of the ENSWM method, the data are divided into two sets of training and test period. The training period starts from 1 September to 30 November 2011, and the test period includes all the days between 1 December 2011 and 26 February 2012. The ensemble forecasts are generated using different configurations of the WRF model. Nine different configurations for the WRF model are used to build members of the system of consciousness. The configurations for the WRF model are summarised in table 1.

\section{Results}

We use some verification methods such as TS and POD for categorical forecasts, and RMSE and ACC for continuous forecasts.

\subsection{Root mean-squared error}

The spatial pattern of RMSE for the 24-hr precipitation forecast in the desired period (1 December 2011 to 26 February 2012) has been illustrated in figure 2. The results show that except the precipitation maxima along the coast of the Caspian Sea in northern Iran, two other maxima (not shown) could be distinguished over the Zagros 


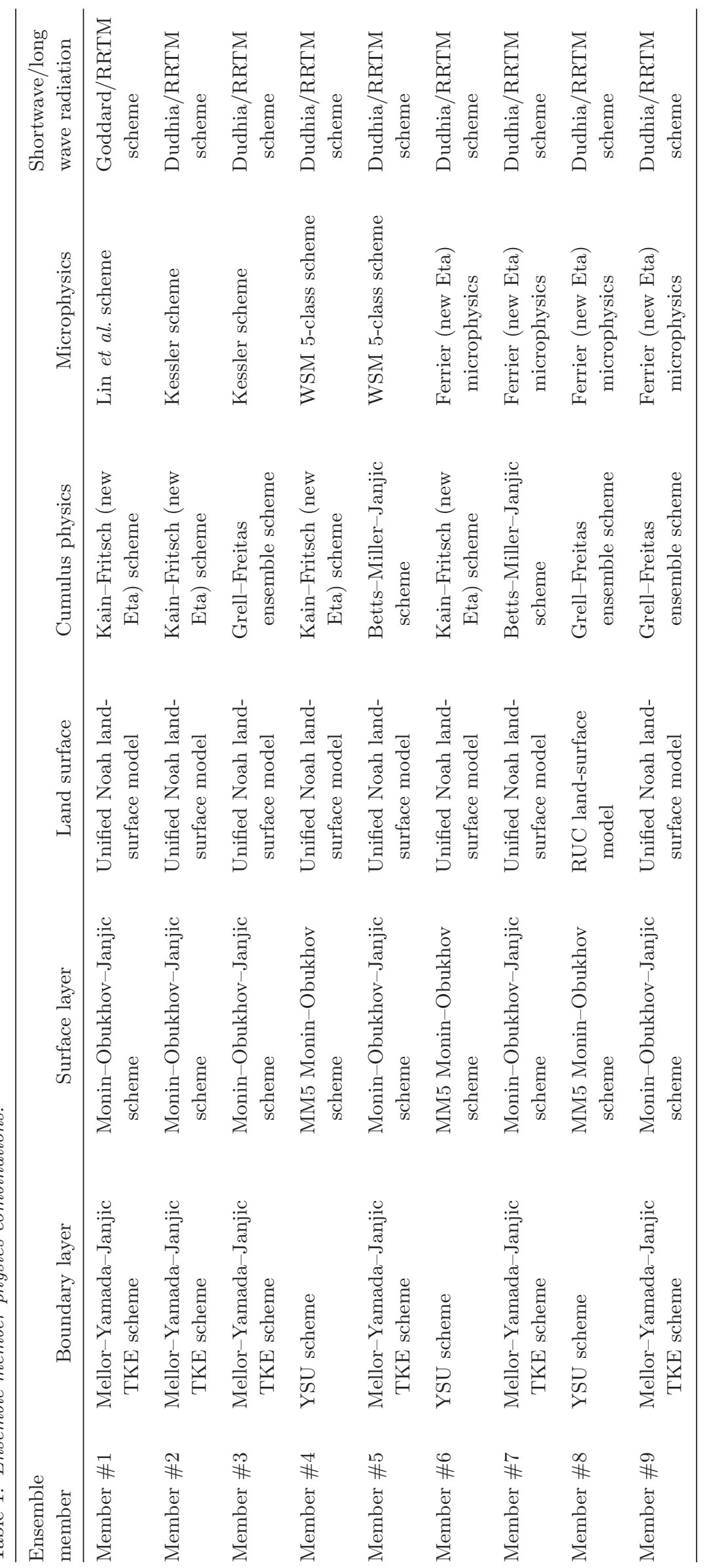




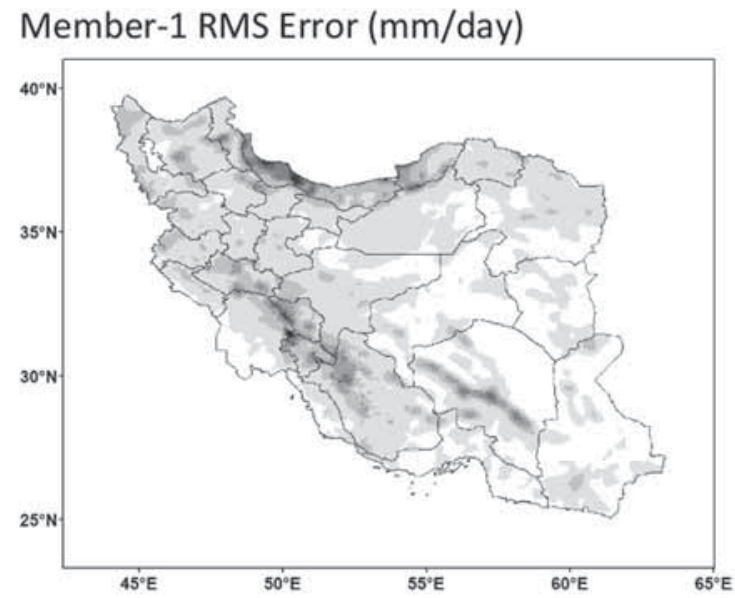

Member-3 RMS Error (mm/day)

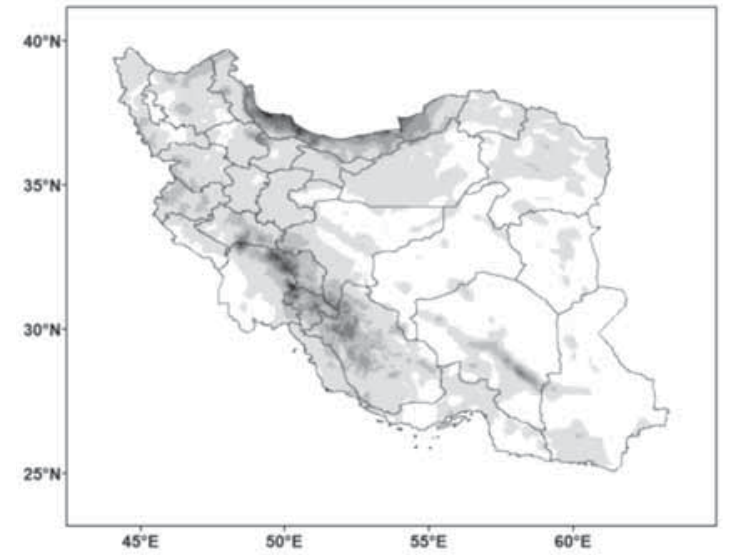

Member-5 RMS Error (mm/day)

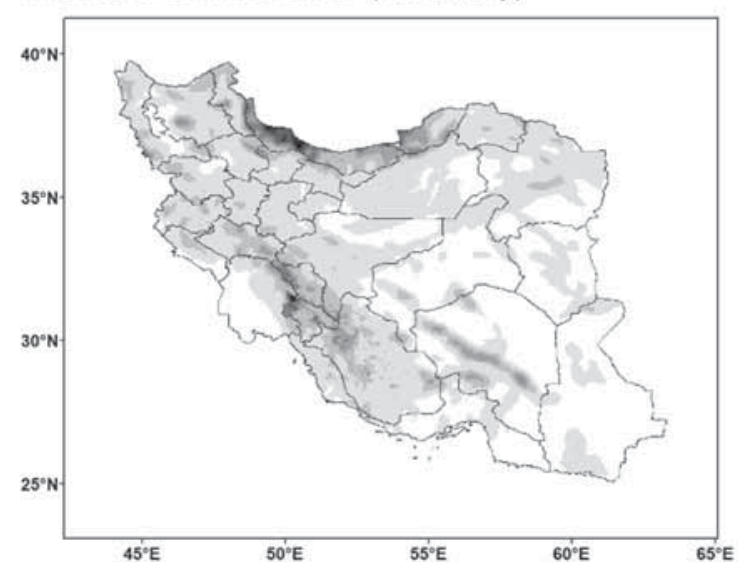

Member-2 RMS Error (mm/day)

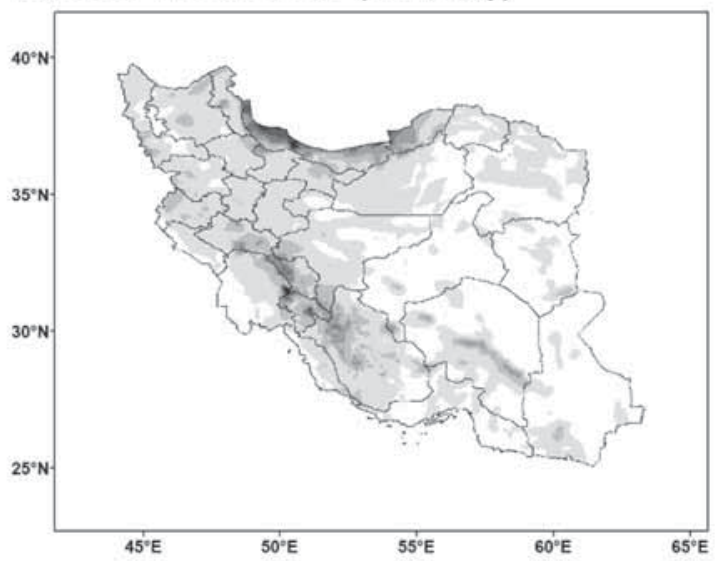

Member-4 RMS Error (mm/day)

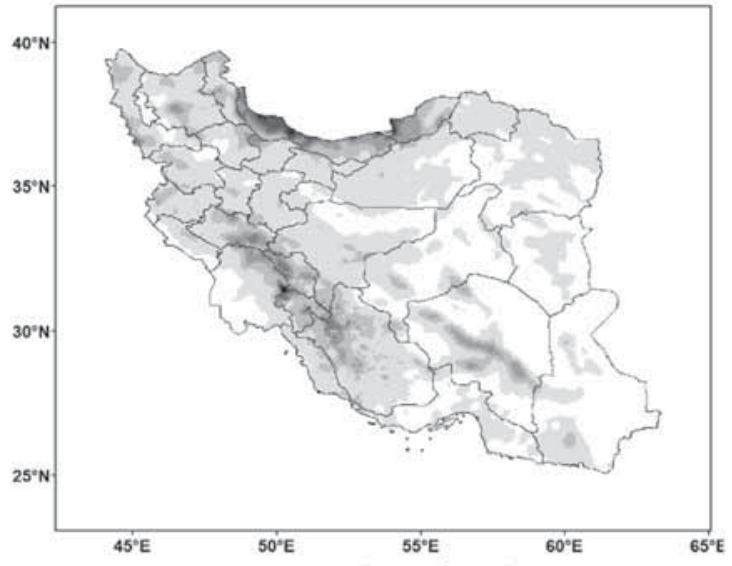

Member-6 RMS Error (mm/day)

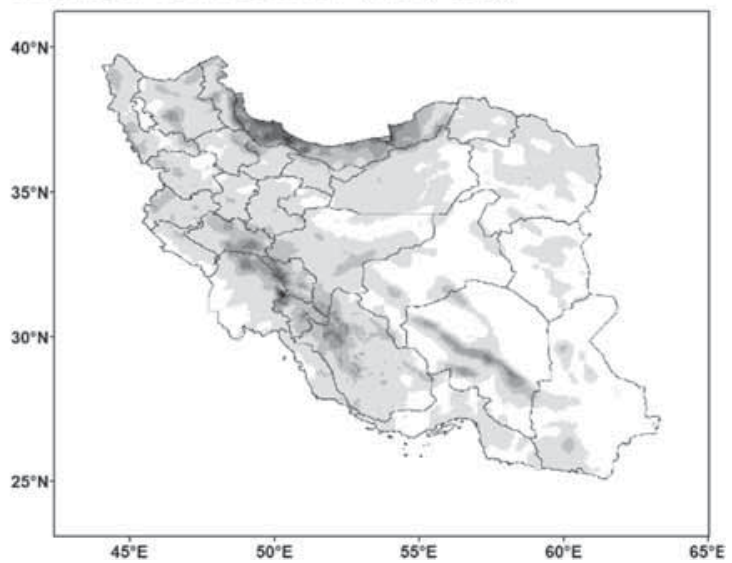

Figure 2. Spatial distribution of RMSE (mm/day) of the ensemble members, ENSM and ENSWM for 24-hr forecasts.

mountain ranges and over the Kerman province (area 28 in figure 1). In the Kerman province, the rainfall maxima are located over the city of Lalehzar and north of Jiroft, such that the maximum is located over the windward of the Haraz mountains.

Figure 2 shows that RMSE almost has the same pattern as precipitation: the higher the precipitation the higher is the RMSE. The three areas, mentioned above, with maximum RMSE, are the main sources of water for the country. So, accurate prediction of rainfall is of critical importance for these areas. For all the members of the ensemble, the highest error is over the Zagros area, in which the RMSE range decreases from 8 to 18 for different ensemble members from 4 to $10 \mathrm{~mm}$ for the ENSWM technique. In the Zagros mountain 

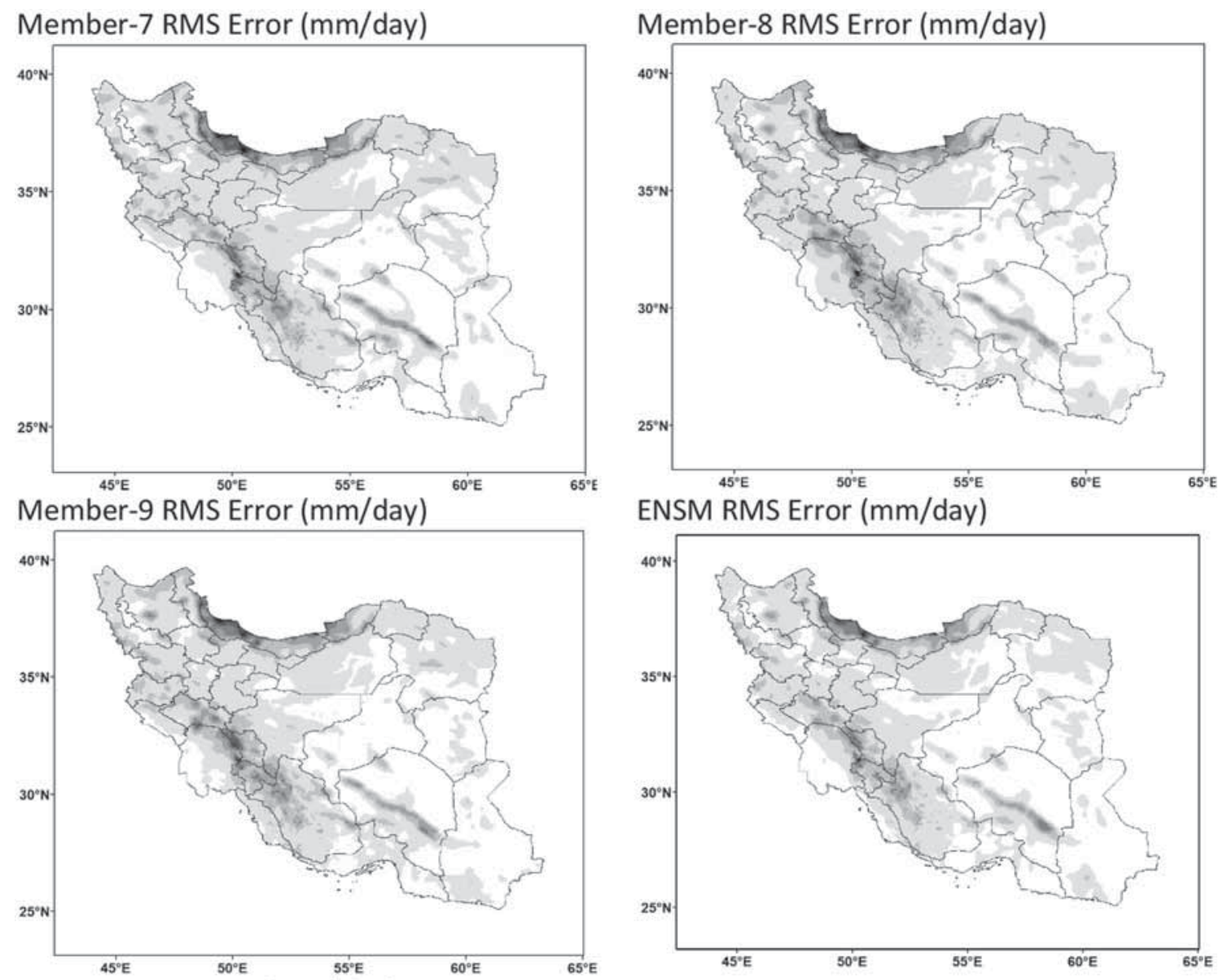

ENSM RMS Error (mm/day)

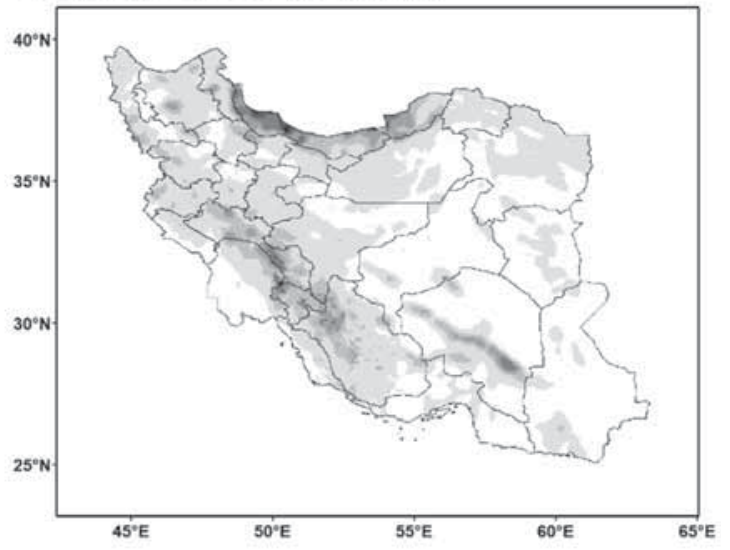

ENSWM RMS Error (mm/day)

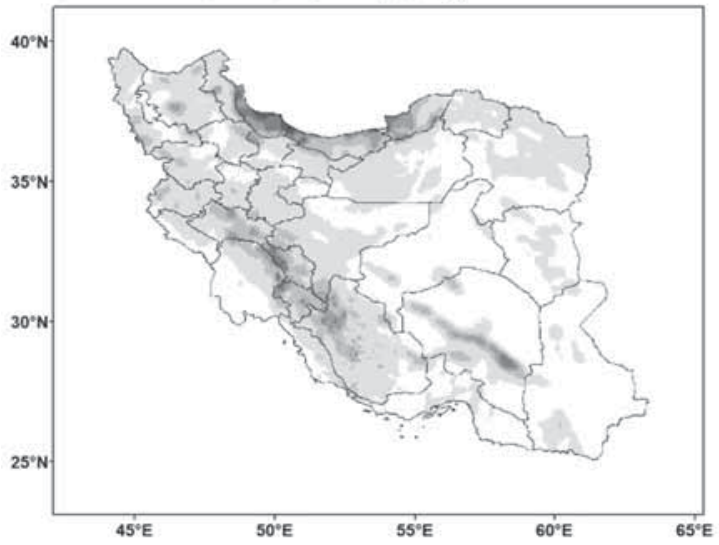

Legend

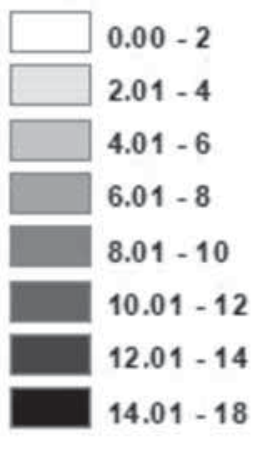

Figure 2. (Continued.)

ranges, there are several peaks with elevation above $4000 \mathrm{~m}$, which are perhaps not properly resolved by the model grid. For example, there is a maximum error over the central areas of the Kerman province that is from 4 to $8 \mathrm{~mm}$. This maximum error is associated with the Haraz peak with elevation of $4500 \mathrm{~m}$. In general, it is seen that over the area with complex topography the impact of statistical post-processing decreases and improvement in the
RMSE of precipitation forecast is not significant compared to the area with homogenous topography.

There is also a maximum error from 2 to $6 \mathrm{~mm}$ in the Tabriz area in the eastern side of Lake Urmia (area 18 in figure 1) which is associated with the Sahand Mountain (area 18 in figure 1) peaks with a height of $>3500 \mathrm{~m}$. The role of Lake Urmia in precipitation is not 
(a) 24-h

RMSE

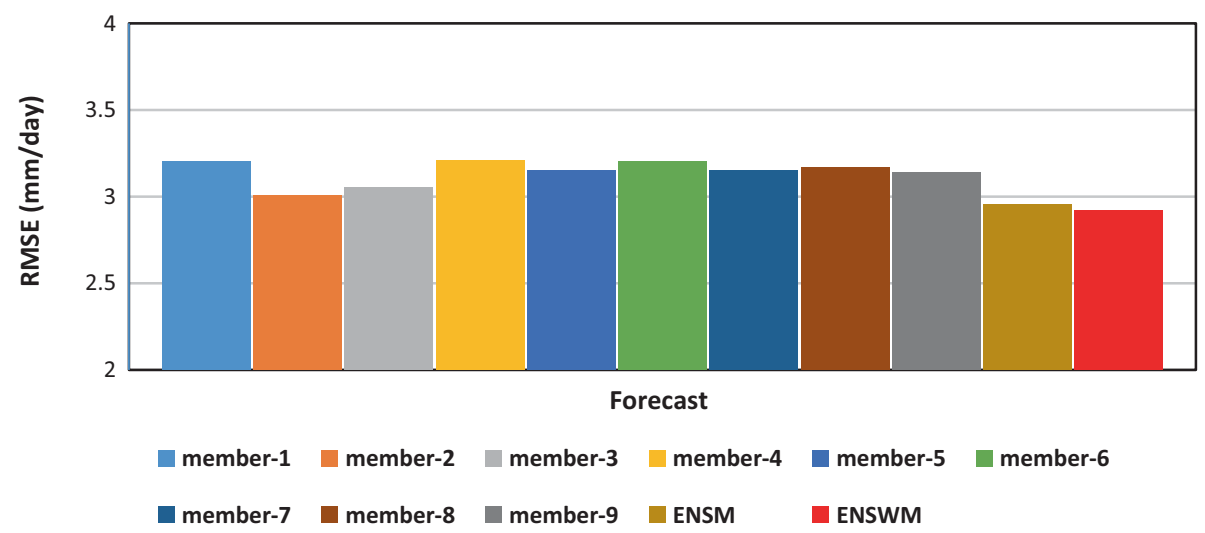

(b) 48-h

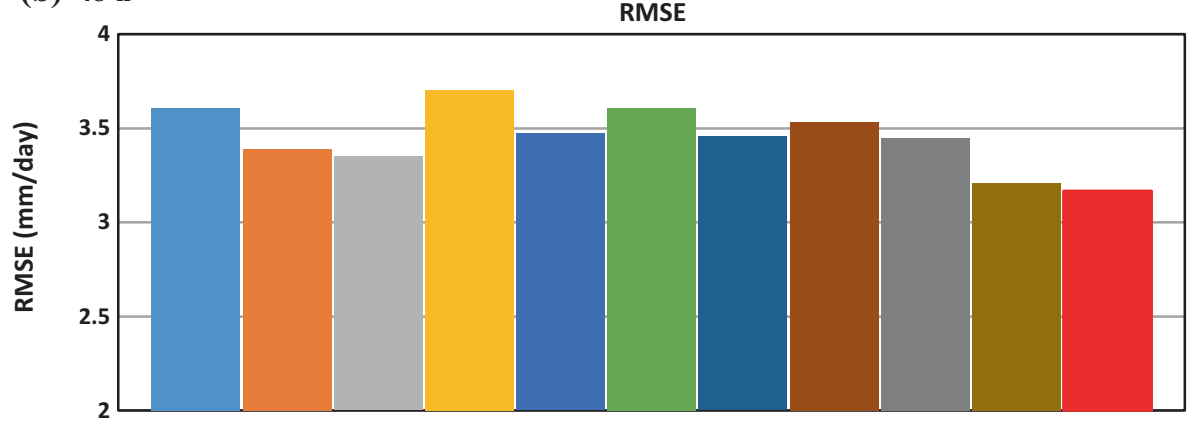

Forecast

member-1 member-2 member-3 member-4 member-5 member-6

member-7 member-8 member-9 ENSM DENSW

(c) $72-\mathrm{h}$

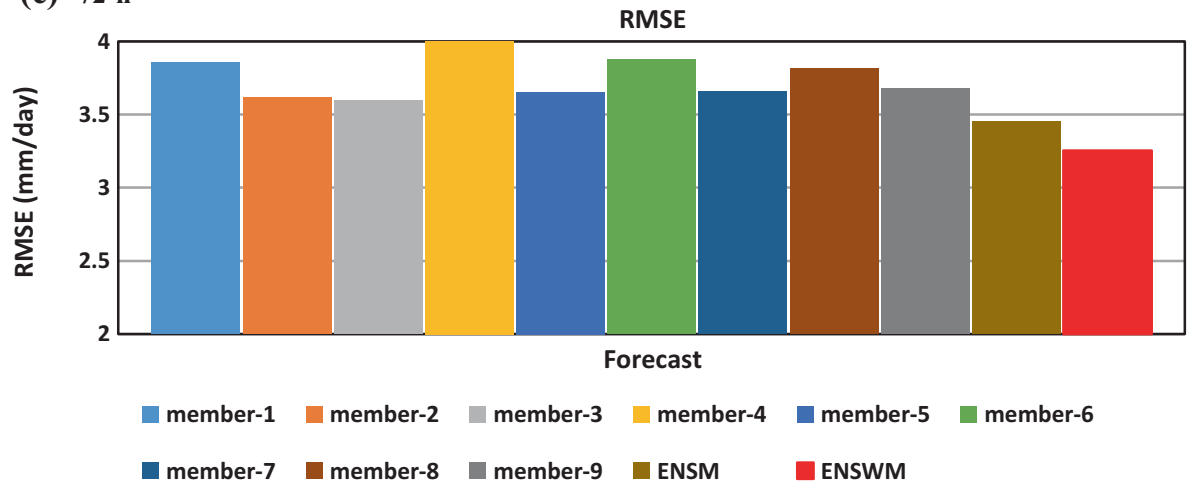

Figure 3. RMSE for the ensemble members, ENSM and ENSWM over the whole country: (a) 24-hr, (b) 48-hr, and (c) 72-hr forecasts.

highlighted in these areas. Mediterranean systems are also effective in producing rainfall in area 18 in figure 1.

For all the ensemble members, the highest error is seen over the northern areas of the country along the coasts of the Caspian Sea. Over this area, the maximum RMSE decreases from $8 \mathrm{~mm}$ for different ensemble members to $12 \mathrm{~mm}$ for the ENSWM technique.

RMSE of the ensemble members, ENSM and ENSWM 24- to 72-hr forecast in comparison to the observations in Iran are given in figure 3. As is seen, ENSWM presents smaller RMSE values. A reduction in error for ENSWM and ENSM indicates 

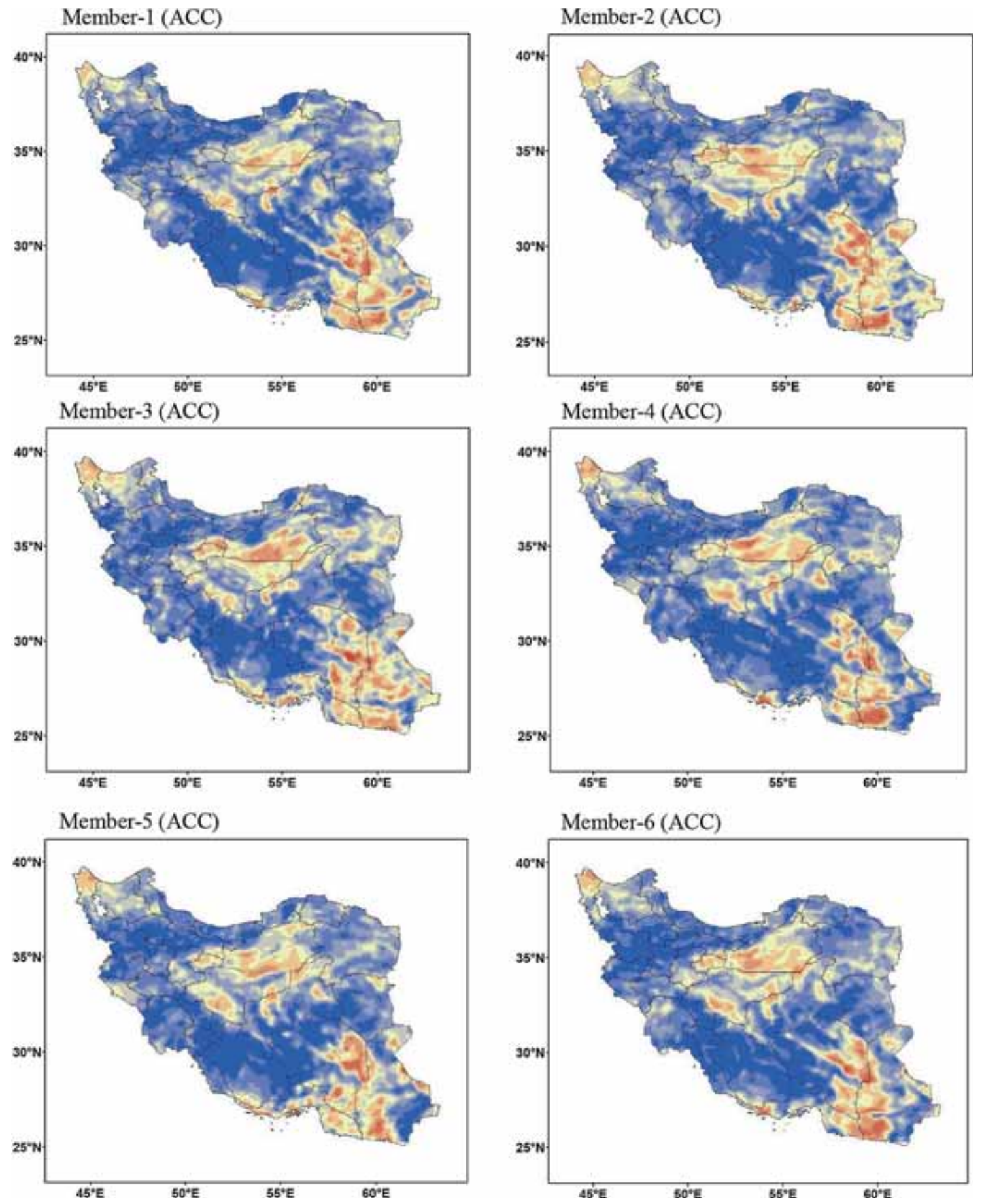

Figure 4. Spatial distribution of season's (1 September 2011 to 30 February 2012) ACC of the ensemble members and MME products for 24-hr forecasts.

that forecast skill is improved using the ENSWM technique as compared to the individual ensemble members.

\subsection{Anomaly $C C$}

ACC, which is the correlation between the forecasts and observations (analysis fields) pairs, is one of the most widely used measures in the verification of spatial fields. For computation of anomaly CC, observed climatology based on 15 yr of gridded reanalysed fields of ERA-Interim data (Dee et al. 2011) was used. If the variation pattern of the anomalies of the forecast is coincident with that of the anomalies of verifying value, ACC will take the maximum value 

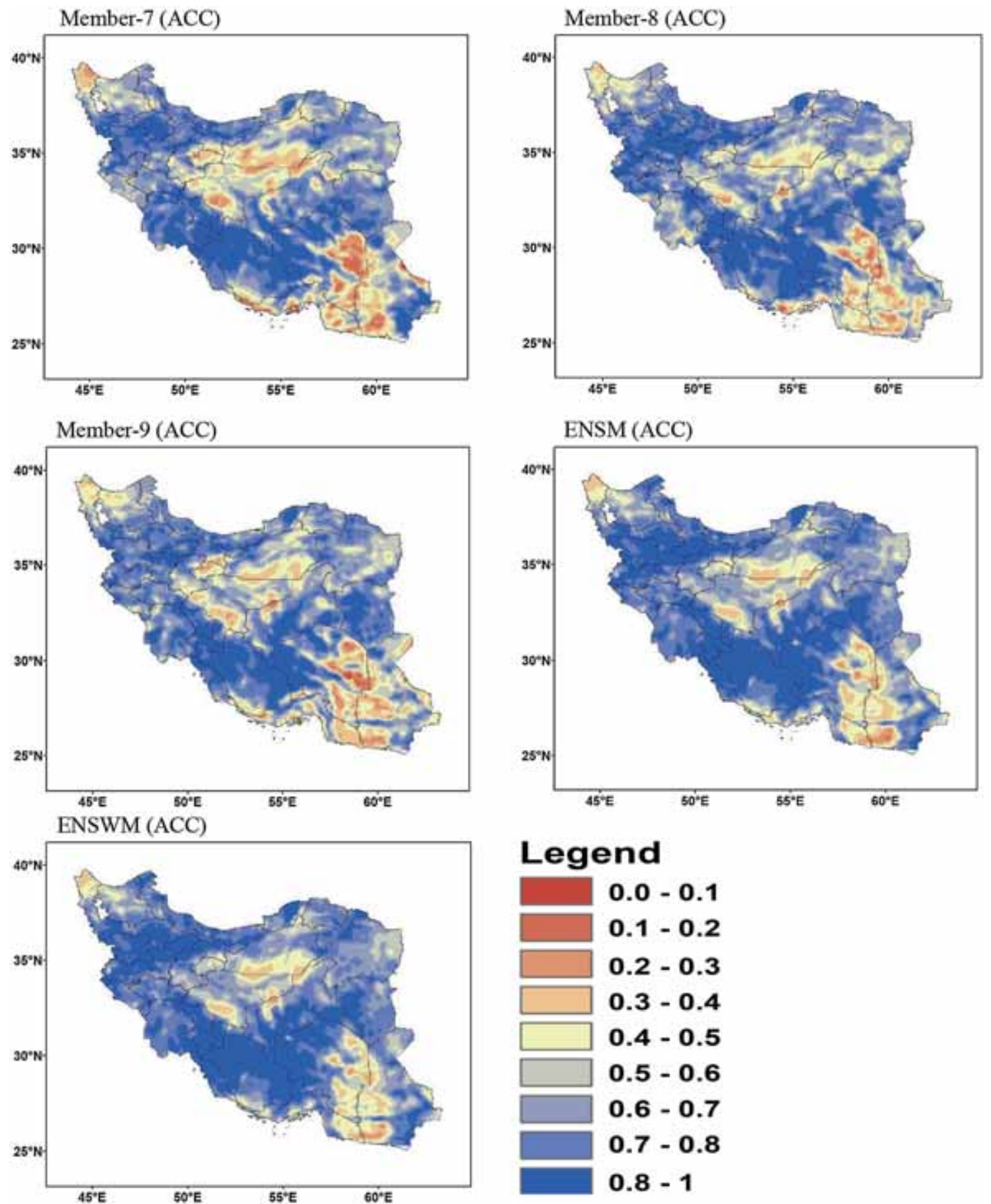

\section{Legend}

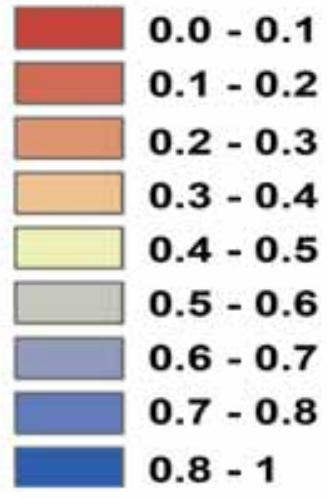

Figure 4. (Continued.)

of 1 . The ACC between the daily domain mean observed and forecasted precipitation for 24-hr forecasts over the country during winter 2011 is calculated and presented in figure 4. As it is seen, the ACC value is, in general, high for all members of the ensemble, but for ENSWM and ENSM, the magnitude of ACC across the country is higher compared to that of the individual ensemble members.

For most parts of the domain, the value of ACC for all ensemble members is from 0.4 to 0.8 , with the exception of some areas in the central, southeast and small areas of the northwest of Iran, where the value of ACC varies from 


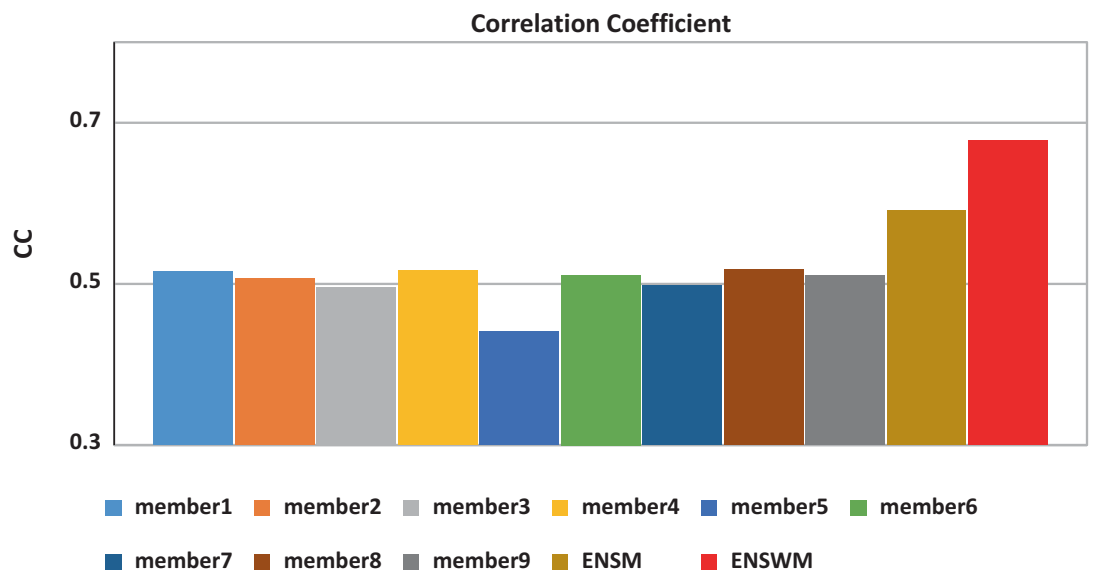

Figure 5. Temporal CC of the ensemble members, ENSM and ENSWM winter 2011 over the whole country.

0.1 to 0.3 . Inter-comparison between ENSWM and ensemble member reveals that ENSWM has slightly higher ACC scores when compared to ENSM and individual ensemble members for the whole region. In the central, southeast and northwest regions of the country, it is from 0.3 to 0.5 and in other parts, the ACC changes from 0.7 to 1.

The northwestern parts of the country are the only areas in which the spring rainfall contribution is higher than what other regions receive. In the northwest, the rainfall drastically varies over the years and consequently, the ACC rates get decreased.

The temporal CC between the 24-hr precipitation forecast and the corresponding observation during winter 2011 is calculated and shown in figure 5. The magnitude of 24-hr for ENSWM and ENSM has higher scores of temporal CC compared to members of the ensemble. The CC lies between 0.44 and 0.67 for ENSWM, ENSM and all members of the ensemble in 24-hr forecast. ENSM and ENSWM have higher scores than members of the ensemble for the 24-hr forecasts.

\subsection{TS and POD for categorical forecasts}

It is worth to examine the skill of precipitation forecasts in terms of precipitation amounts in different thresholds. So, the common statistical measures including TS or critical success index and POD or hit rate (HR) are computed for the comparison in different thresholds of precipitation amounts.
To assess the model performance in different rainfall thresholds, the TS and HR are calculated for four thresholds of $0.1,5,15$ and $25 \mathrm{~mm}$. The results are presented in figures 6 and 7 . TS of the above-mentioned rainfall thresholds for individual ensemble members, ENSM and ENSWM over Iran for 24- to 72-hr forecast is shown in figure 6 . Higher value of TS indicates better forecast with a theoretical limit of 1.0 for a perfect forecast. As seen in figure 6, the value of TS decreases with increasing the threshold, such that the highest and lowest values of TS are associated with 0.1 and $25 \mathrm{~mm}$ thresholds, respectively. In addition, it is seen that ENSM and ENSWM have higher value of TS compared to all individual ensemble members. It is seen that the TS skill score of the 24-hr forecast for ENSWM begins from 0.88 and then becomes 0.54 at the $5 \mathrm{~mm}$ rain threshold, 0.41 at $15 \mathrm{~mm}$ rain threshold and becomes 0.32 at $25 \mathrm{~mm}$ rain threshold, respectively (figure 6). TS of all ensemble members looks similar for all the forecast thresholds. However, in general, TS of ENSWM is slightly higher than ENSM and other ensemble members for all thresholds. Two other skill scores, HR or POD, are the ratio of the number of the correct forecasts for an occurrence of precipitation above a threshold to the total number of observations of the rainfall concerned. HR for rainfall threshold of $0.1,5,15$ and $25 \mathrm{~mm} /$ day of the ensemble members, ENSM and ENSWM over Iran for 24- to 72-hr forecast is shown in figure 7. For HR, the ENSM and ENSWM products are mostly seen to perform much better than the ensemble members in all thresholds. ENSWM shows higher values of HR skill than ENSM and other ensemble 
(a) 24-h
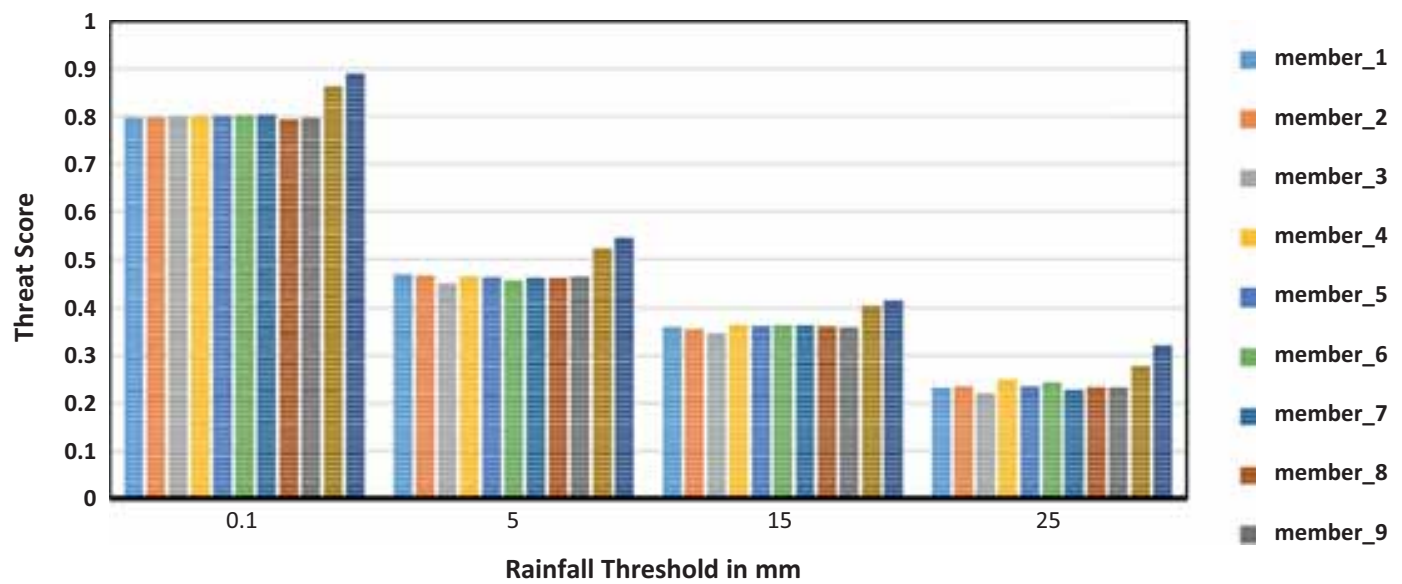

(b) $48-h$
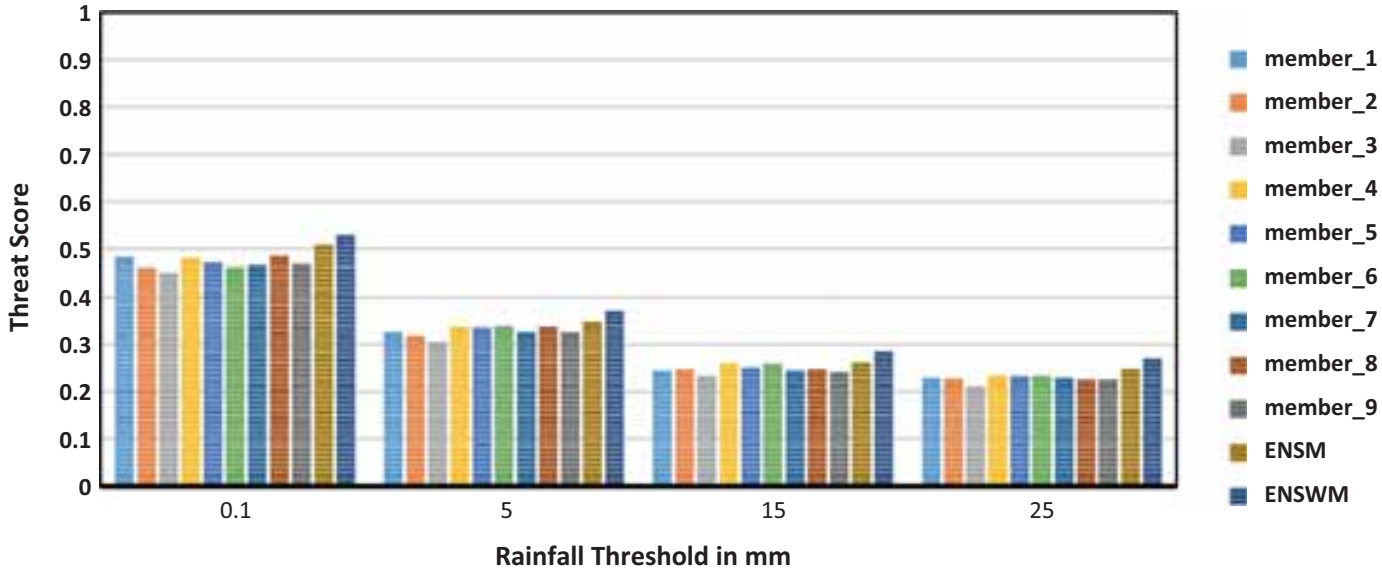

(c) $72-\mathrm{h}$

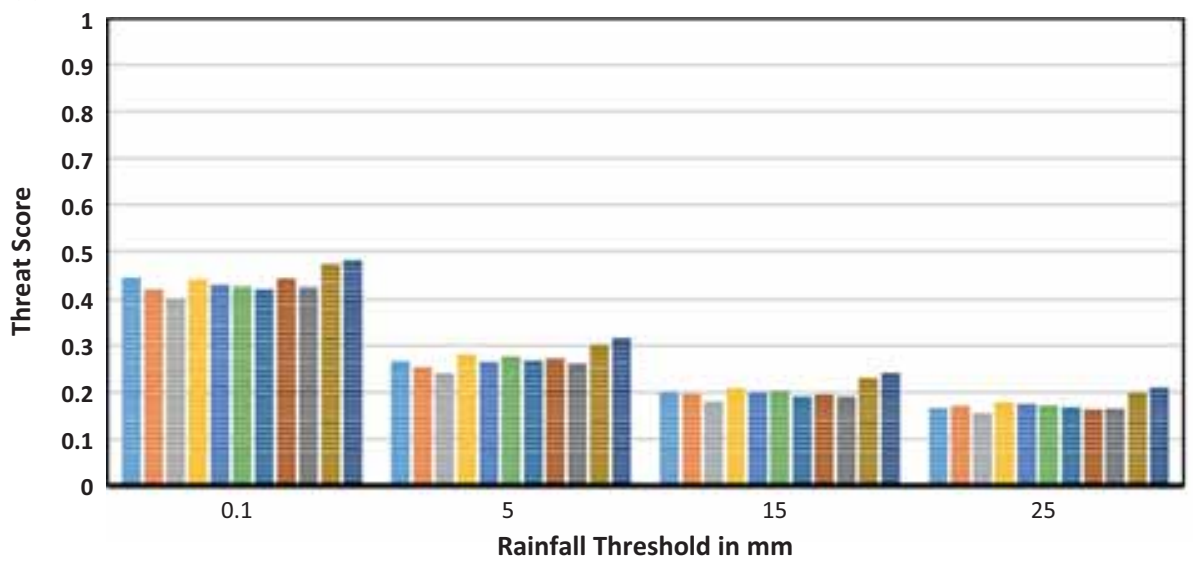

in member_1
$=$ member_2
in member_3
a member_4
= member_5
$=$ member_6
$=$ member_7
$=$ member_8
$=$ member_9

Figure 6. TS for (a) 24-hr, (b) 48-hr and (c) 72-hr forecast of the ensemble members, ENSM and ENSWM during winter 2011 across the country.

members for all days and for all thresholds across the country. In general, the values of $\mathrm{HR}$ for 24-, 48- and 72-hr forecasts of ENSWM have higher scores compared to ENSM and member models.
Figure 8 shows the time series of countryaveraged daily precipitation for the period starting from 1 September 2011 to 26 February 2012 based on the ensemble members in (a) and ENSWM in (b) along with the observed rainfall. The time series 
(a) 24-h
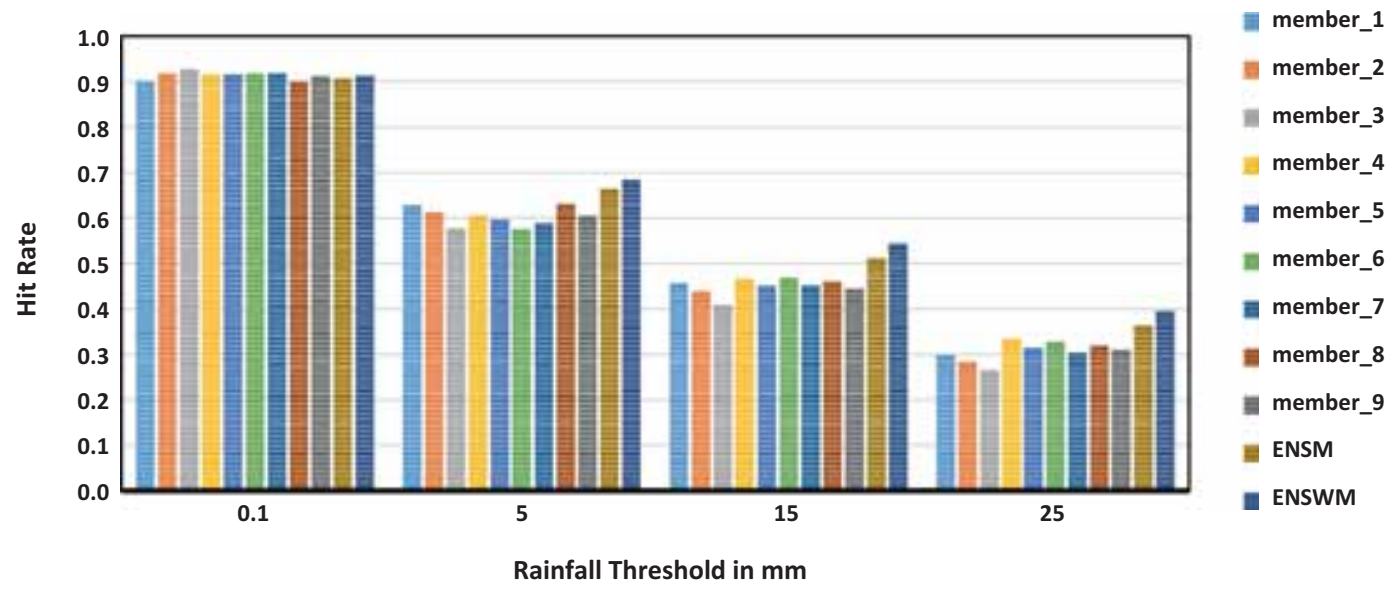

(b) 48-h
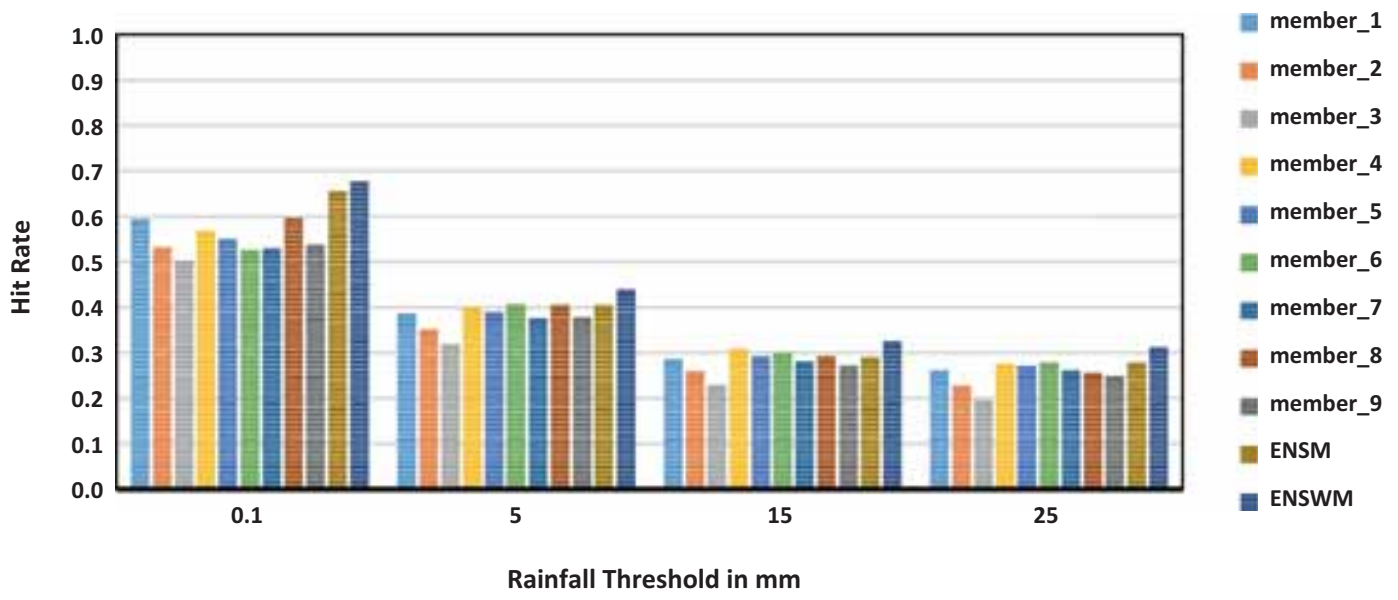

(c) $72-\mathrm{h}$
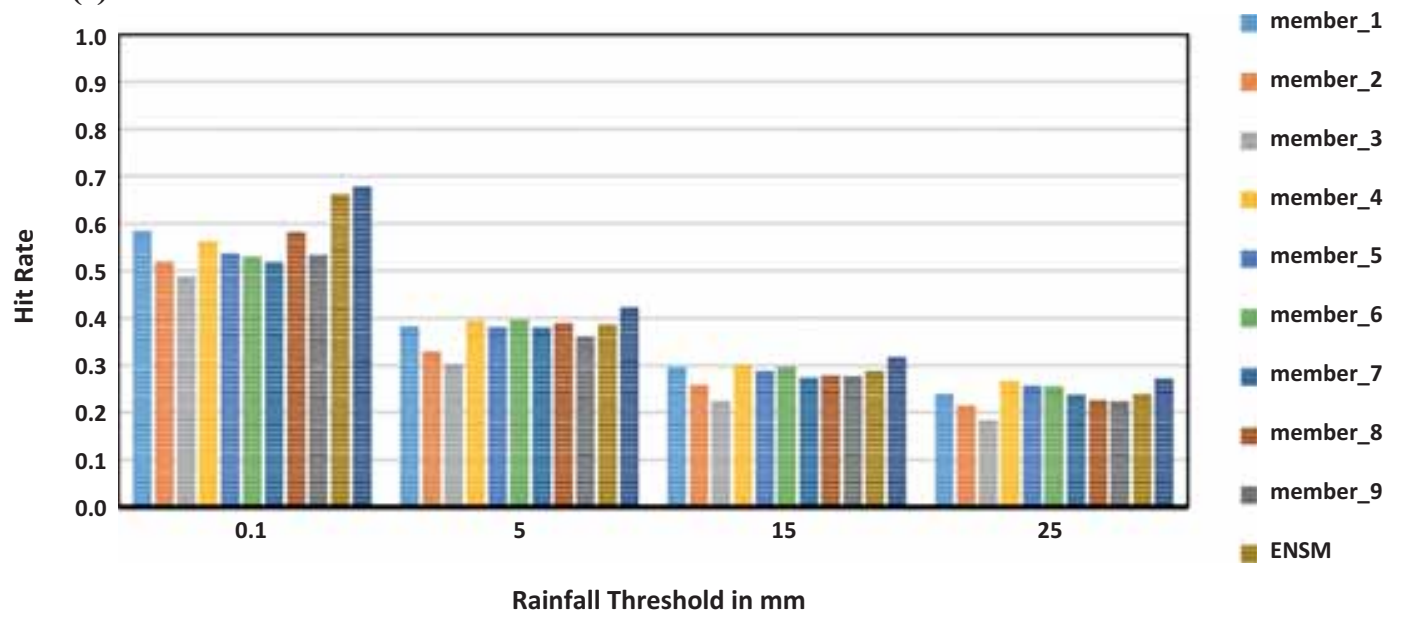

Figure 7. HR for (a) 24-hr, (b) 48-hr and (c) 72-hr forecast of the ensemble members, ENSM and ENSWM during winter 2011 across the country.

indicates that although the ensemble, in general, is able to take daily ups and downs of rainfall spells, it significantly under-estimates the rainfall amount.
This also shows how the efficiency of the individual member influences the performance of the ensemble forecast. 

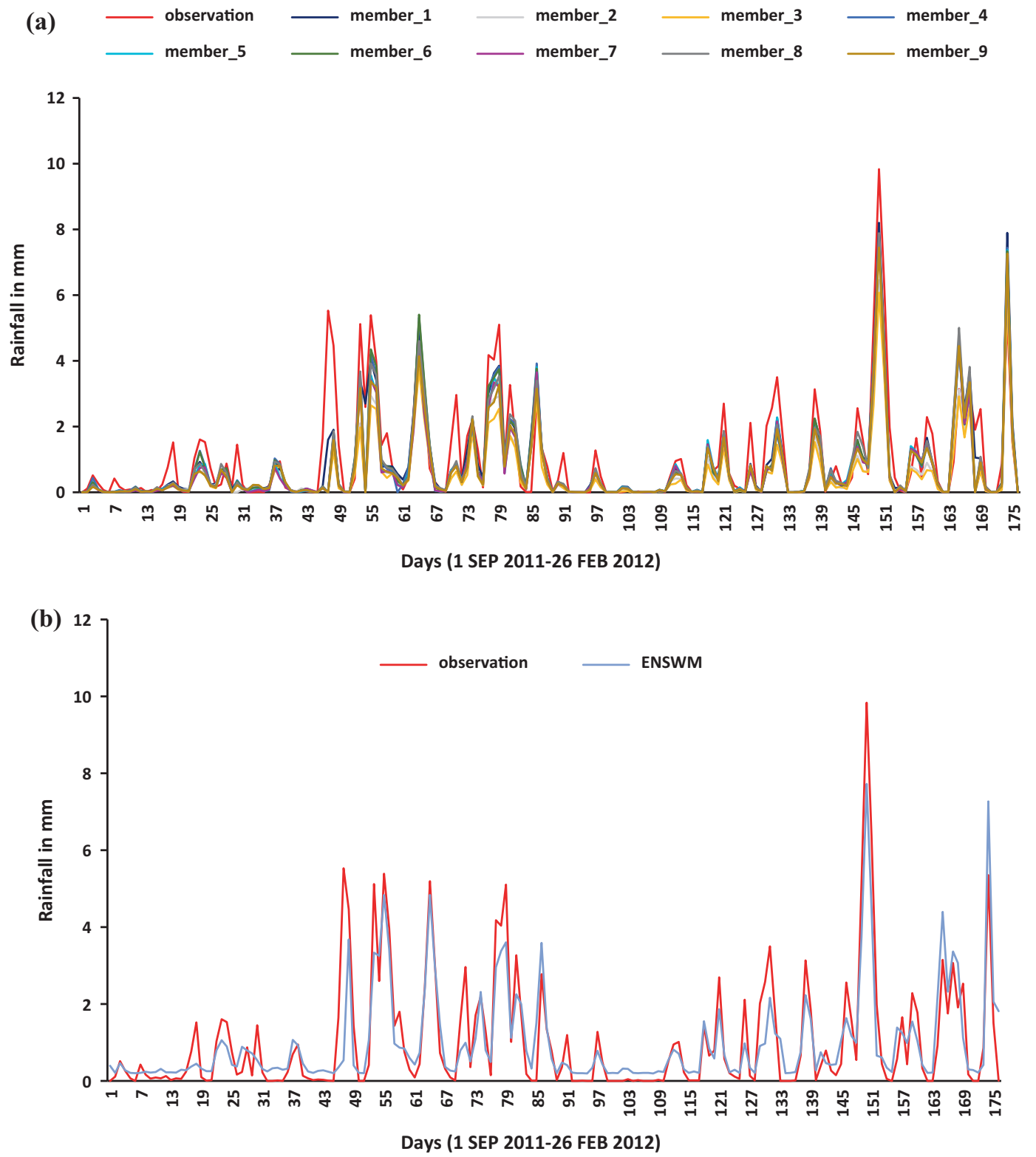

Figure 8. Time series of spatial averaged daily precipitation observed and predicted ( $\mathrm{mm}$ ) by (a) different member ensemble and (b) the ENSWM across the whole country (land only) based on 24-hr forecasts for the time period 1 September 2011 to 26 February 2012.

\section{Conclusions}

The study provides a concise documentation of the current level of skill of the ENSWM technique used for forecasting daily precipitation over Iran in the short-range time scale during winter of 2011-2012.

The WRF model has been used to design a multi-physics ensemble that comprises nine model configurations with different cumulus, PBL, microphysics and parameterisation schemes of the surface layer. The weight for each of the member of the ensemble at each grid point is assigned on the basis of past performance of the ensemble member during a training time period. Apart from ENSWM, a simple ENSM forecast is also generated and experimented.

The comparison of rainfall prediction skill of the ENSWM forecast against the ensemble members reveals that the ENSWM is able to provide more accurate forecast of rainfall over Iran. The calculated values of RMSE and CC clearly indicate that ENSWM forecast is superior to the forecast of the individual member of ensemble and ENSM. In terms of rainfall amounts, the skill of rainfall forecast for different thresholds over Iran indicates 
that ENSWM produces better skill compared to ENSM and members of the ensemble.

The areas with high mountain ranges of Alborz (in the north) and Zagros (in the west) are important for water resources. On the other hand, the results showed that model errors are generally higher at high altitude mountain ranges. It is thus expected that increasing the model resolution should lead to consistent error reduction.

In general, application of the post-processing leads to consistent error reduction. Such that in the northern Iran along the coasts of the Caspian Sea, the maximum RMSE in the ENSWM and ENSM methods decreased by 30 and $20 \%$, respectively. In the western Iran over the Zagross mountain ranges, the maximum RMSE was decreased by 42 and $30 \%$ in the ENSWM and ENSM methods, respectively.

Ensemble forecasts are computationally expensive to produce compared to the deterministic model forecasts. But considering the fact that except the coastal areas of the Caspian Sea in northern Iran, most of the model errors are associated with mountainous areas, which are the main source of water for the country, producing and using expensive ensemble forecasts are justified.

In other research studies, more sophisticated post-processing techniques such as logistic regression (Wilks 2006), BMA (Raftery et al. 2005) and analogue ensemble (Delle Monache et al. 2013), which are suggested for future works, have been used and experimented with success.

\section{Acknowledgement}

We would like to show our gratitude to IR of Iran Meteorological Organization (IRIMO) for providing access to the observation data.

\section{References}

Buizza R and Palmer T N 1995 The singular-vector structure of the atmospheric global circulation; J. Atmos. Sci. 52 $1434-1456$

Christensen H M, Moroz I M and Palmer T N 2015 Stochastic and perturbed parameter representations of model uncertainty in convection parameterization; J. Atmos. Sci. 72 2525-2544.

Dee D P, Uppala S M, Simmons A J, Berrisford P, Poli P, Kobayashi S, Andrae U, Balmaseda M A, Balsamo G, Bauer P, Bechtold P, Beljaars A C M, van de Berg L, Bidlot J, Bormann N, Delsol C, Dragani R, Fuentes M, Geer A J, Haimberger L, Healy S B, Hersbach H, Hólm E V, Isaksen L, Kållberg P, Köhler M, Matricardi M, McNally A P, Monge-Sanz B M, Morcrette J-J, Park B-K,
Peubey C, de Rosnay P, Tavolato C, Thépaut J-N and Vitart F 2011 The ERA-interim reanalysis: Configuration and performance of the data assimilation system; Quart. J. Roy. Meteorol. Soc. 137 553-597.

Delle Monache L, Eckel T, Rife D and Nagarajan B 2013 Probabilistic weather prediction with an analog ensemble; Mon. Weather Rev. 141 3498-3516.

Durai V R and Bhardwaj R 2013 Improving precipitation forecasts skill over India using a multi-model ensemble technique; Geofizika 30(2) 119-141.

Ebert E E 2001 Ability of a poor man's ensemble to predict the probability and distribution of precipitation; Mon. Weather Rev. 129 2461-2480.

Epstein E S 1969 Stochastic dynamic prediction; Tellus 6 739-759.

Evans R E, Harrison M S J, Graham R J and Mylne K R 2000 Joint medium range ensembles from the Met. Office and ECMWF system; Mon. Weather Rev. 128 3104-3127.

Frogner I-L and Iversen T 2002 High-resolution limited-area ensemble predictions based on low resolution targeted singular vectors; Quart. J. Roy. Meteorol. Soc. $1281321-$ 1341.

Houtekamer P L, Lefaivre L, Derome J, Ritchie H and Mitchell H L 1996 A system simulation approach to ensemble prediction; Mon. Weather Rev. 124 1225-1242.

Jaiswal N, Kishtawal C M and Bhomia S 2018 Similaritybased multi-model ensemble approach for 1-15-day advance prediction of monsoon rainfall over India; Theor. Appl. Climatol. 132 639-645.

Johnson C and Swinbank R 2009 Medium-range multi-model ensemble combination and calibration; Quart. J. Roy. Meteorol. Soc. 135 777-794.

Krishnamurti T N, Kishtawal C M, Larow T, Bachiochi D, Zhang Z, Willford E C, Gadgil S and Surendran S 1999 Improved weather and seasonal climate forecasts from multimodel superensemble; Science 2851548 1550 .

Krishnamurti T N, Kishtawal C M and Zhang Z et al. 2000 Multi-model ensemble forecasts for weather and seasonal climate; J. Clim. 13(23) 4196-4216.

Krishnamurti T N, Mishra A K, Chakraborty A and Rajeevan M 2009 Improving global model precipitation forecasts over India using downscaling and the FSU superensemble. Part I: 1-5-day forecasts; Mon. Weather Rev. 137 2713-2735.

Kumar A, Mitra A K, Bohra A K, Iyengar G R and Durai V R 2012 Multi-model ensemble (MME) prediction of rainfall using neural networks during monsoon season in India; Meteorol. Appl. 19 161-169.

Leith C E 1974 Theoretical skill of Monte Carlo forecasts; Mon. Weather Rev. 102 409-418.

Masters T 1993 Practical Neural Network Recipes in $C_{++}$, Academic Press, San Diego, CA, 493p.

Mishra A K and Krishnamurti T N 2007 Current status of multi-model super-ensemble operational NWP forecast of the Indian summer monsoon; J. Earth Syst. Sci. 116(5) $1-16$

Mitra A K, Iyengar G R, Durai V R, Sanjay J, Krishnamurti T N, Mishra A and Sikka D R 2011 Experimental realtime multi-model ensemble (MME) prediction of rainfall during monsoon 2008: Large-scale medium-range aspects; J. Earth Syst. Sci. 120(1) 1-22. 
Molteni F, Buizza R, Palmer T N and Petroliagis T 1996 The ECMWF ensemble prediction system: Methodology and validation; Quart. J. Roy. Meteorol. Soc. 122 73119.

Muller B and Reinhardt J 1991 Neural Networks: An Introduction, the Physics of Neural Networks Series, SpringerVerlag, Berlin, 266p.

Nalder I A and Wein R W 1998 Spatial interpolation of climatic normals: Test of a new method in the Canadian boreal forest; Agric. Forest Meteorol. 92 211-225.

Raftery A E, Gneiting T, Balabdaoui F and Polakowski M 2005 Using Bayesian model averaging to calibrate forecast ensembles; Mon. Weather Rev. 133 1155-1174.

Richardson D S 2001 Ensembles using multiple models and analyses; Quart. J. Roy. Meteorol. Soc. 127 18471864.

Romine G S, Schwartz C S, Berner J, Fossell K R, Snyder C, Anderson J L and Weisman M L 2014 Representing forecast error in a convection-permitting ensemble system; Mon. Weather Rev. 142 4519-4541.

Roy Bhowmik S K and Durai V R 2008 Multimodel ensemble forecasting of rainfall over Indian monsoon region; Atmosfera 21(3) 225-239.

Roy Bhowmik S K and Durai V R 2010 Application of multimodel ensemble techniques for real time district level rainfall forecasts in short range time scale over Indian region; Meteorol. Atmos. Phys. 106(1-2) 19-35.

Roy Bhowmik S K and Durai V R 2012 Development of multimodel ensemble based district level medium range rainfall forecast system for Indian region; J. Earth Syst. Sci. $121273-285$.

Soltani M, Laux P, Kunstmann H, Stan K, Sohrabi M M, Molanejad M and Zawar-Reza P 2016 Assessment of climate variations in temperature and precipitation extreme events over Iran; Theor. Appl. Climatol. 126(3-4) 775795 .

Toth Z and Kalnay E 1993 Ensemble forecasting at NMC: The generation of perturbations; Bull. Am. Meteor. Soc. 74 2317-2330.

Toth Z and Kalnay E 1997 Ensemble forecasting at NCEP and the breeding method; Mon. Weather Rev. 125 32973319.

Wang X and Bishop C H 2003 A comparison of breeding and ensemble transform Kalman filter ensemble forecast schemes; J. Atmos. Sci. 60 1140-1158.

Wang X, Bishop C H and Julier S J 2004 Which is better, an ensemble of positive-negative pairs or a centered spherical simplex ensemble? Mon. Weather Rev. 132 1590-1605.

Wilks D S 2006 Statistical Methods in the Atmospheric Sciences; 2nd edn, Academic Press, New York, 627p. 